\title{
Giomeros en Odontopediatría. Revisión narrativa
}

\section{Artículo de Revisión}

Rocío Meza Salcedo 1,a, Alan Pérez Valverde 1,b

1 Universidad Nacional de San Antonio Abal del Cusco, Facultad de Ciencias de la Salud, Cusco, Perú.

${ }^{a}$ Magíster en Políticas y Gestión en Salud. Especialista en Odontopediatría.

${ }^{b}$ Cirujano Dentista.

\section{Correspondencia:}

Rocío Meza Salcedo: mezasalcedorocio@gmail.com Av. Camino Real 110. Urb. Vallecito. Cusco Perú. ORCID: 0000-0003-0538-9177

\section{Coautor:}

Alan Pérez Valverde: alanpv1983@gmail.com ORCID: 0000-0002-9476-3548

\section{Gyomers in Pediatric Dentistry. Narrative review}

\section{Editora:}

Antonieta Pérez-Flores

Universidad de Concepción, Chile.

Conflicto de intereses: ninguno.

Fuente de financiamiento: autofinanciado.

Recibido: 30/04/20

Aceptado: $14 / 09 / 20$

Publicado: 16/11/20

\section{Resumen}

La presente revisión tiene por objetivo comparar las ventajas que otorgan los giomeros en Odontopediatría frente a otros cementos dentales. Se realizó una revisión narrativa en las bases de datos SciELO y en los buscadores bibliográficos PubMed y Google Schoolar, hasta el 2019. De los 43 artículos se excluyeron ocho ya que no presentaban información con resultados relevantes, quedando así un total de 35 artículos para nuestra investigación. Se obtuvieron resultados comparativos, en cuanto a la recarga o liberación de flúor, fuerza de cizallamiento, estabilidad de color y comportamiento clínico en la superficie dentaria; encontrándose que el giomero es un material liberador de flúor por excelencia, así como por su adhesión a la dentina cumpliendo con la aceptación y directrices de la dentina y esmalte del programa de Materiales Adhesivos establecidos por la Asociación Dental Americana.

Palabras clave: Compómeros; Materiales dentales; Restauración dental permanente (fuente: DeCS BIREME).

\begin{abstract}
The purpose of this review is to compare the advantages of giomers in pediatric dentistry compared to other dental cements. A narrative review was performed in the SciELO databases and in bibliographic search engines PubMed, Google Scholar, until 2019. Of the 43 articles, eight were excluded since they did not present information with relevant results, leaving a total of 35 articles for our investigation. Comparative results were obtained in terms of fluoride recharge or release, shear force, color stability and clinical behavior on the tooth surface; finding that giomers are fluoride-releasing materials par excellence, as well as for its adhesion to dentin, complying with the acceptance and guidelines of dentin and enamel of the Adhesive Materials program established by the American Dental Association.
\end{abstract}

Keywords: Compomers; Dental materials; Dental restoration, permanent (source: MeSH NLM).

(c) Los autores. Este artículo es publicado por la revista Odontología Sanmarquina de la Facultad de Odontología, Universidad Nacional Mayor de San Marcos. Este es un artículo de acceso abierto, distribuido bajo los términos de la licencia Creative Commons Atribucion - No Comercia_Compartir Igual 4.0 Internacional. (http://creativecommons.org/licenses/by-nc-sa/4.0/) que permite el uso no comercial, distribución y reproducción en cualquier medio, siempre que la obra original sea debidamente citada. 


\section{Introducción}

La boca es de vital importancia, pues es uno de los órganos principales del sistema digestivo. En este sentido, la carencia de salud bucal trae consecuencias que afectan la calidad de vida del paciente no solamente a nivel psicosocial, debido a su falta de estética, sino también biológicamente debido al dolor y malestar al comer ${ }^{1,2}$.

Justamente, las caries son una de las enfermedades bucodentales más frecuentes en la población mundial. La Organización Mundial de la Salud (OMS) afirma que "el 60\%-90\% de los escolares y casi el 100\% de los adultos tienen caries dental en todo el mundo" ${ }^{3}$.

Ya con Wilson y Kent que introdujeron los ionómeros de vidrio ${ }^{4}$ y el Dr. Bowen al incorporar las resinas compuestas ${ }^{5}$ influenciaron en la revolución de los materiales dentales; logrando bloquear las lesiones de caries antes que se produzca la cavitación, logrando aumentar la resistencia de los dientes ante los ácidos, produciendo un mecanismo de remineralización en la superficie del esmalte $^{6}$.

El término giomeros proviene de glass ionomer (ionómero vítreo) + composite. Los giomeros fluidos, son una tecnología relativamente nueva, que emplea el ionómero de vidrio previamente reaccionado y resina compuesta, dentro de su composición con nanotecnología 7,8, lo que permite obtener las propiedades de ambos ${ }^{9}$ : del ionómero de vidrio, la liberación de iones de flúor que proporciona un efecto anticariogénico para su absorción por el esmalte y dentina y recarga de flúor ${ }^{6}$; y de la resina, la estética, pulibilidad ${ }^{10,11}$ la fácil manipulación y sus propiedades físicas de los composites híbridos al utilizarlos juntos ${ }^{12}$.

Se ha comprobado que los giomeros pueden ser utilizados en superficies oclusales y proximales, con propiedades de manejo mejoradas, facilitando la reconstrucción de dientes posteriores ${ }^{13}$; sin embargo, existen algunas desventajas, tales como la contracción de polimerización, la microfiltración y la irritación pulpar. ${ }^{14}$ Para ello McLean en 1985 introdujo la "técnica de emparedado" o también conocida como restauración de laminado, que combina ambos materiales reduciendo la microfiltración en los márgenes de restauración ${ }^{14,15}$ aprovechando al máximo sus propiedades físicas y estéticas. ${ }^{16,17}$

Por ello el objetivo de este estudio fue hallar conclusiones en cuanto a la recarga o liberación de flúor, fuerza de cizallamiento, estabilidad de color y comportamiento clínico en la superficie dentaria del giomero en comparación con otros cementos convencionales usados en Odontopediatría.

\section{Metodología}

Se realizó una revisión narrativa de las bases de datos SciELO y en los buscadores bibliográficos PubMed y Google Schoolar hasta el 2019, para tener un mayor rango de años de búsqueda de información y para contrastar resultados de las diferentes investigaciones a lo largo de estos años; utilizando las siguientes palabras clave: compómeros, resinas compuestas, restauración dental permanente. De los 43 artículos obtenidos en su mayoría en inglés se excluyeron ocho ya que no cumplían con los criterios de inclusión al no presentar información con resultados relevantes, también se excluyeron reportes de casos quedando así un total de 35 artículos para nuestra investigación.

\section{Revisión de la literatura actual}

En los estudios investigados se tomaron en cuenta distintos parámetros, entre ellos:

Recarga o liberación de flúor. El relleno S-PRG que contienen los giomeros, tienen la propiedad de liberar y recargar flúor dependiendo de la concentración de este en la boca y por presentar mayor liberación inicial de fluoruro son los que presentan mayor capacidad de esta propiedad ${ }^{18}$, potenciados por una mayor concentración (5000 ppm) de fluoruros tópicos y un mayor número de exposiciones diarias (mínimo 2 veces al día). ${ }^{19}$

La fuerza y estabilidad del composite permanece inalterada y la liberación de flúor ocurre exactamente donde el efecto puede desarrollarse mejor ${ }^{20}$. Esto significa que las áreas de contacto adyacentes de la estructura dura natural del diente están protegidas de forma efectiva de la caries debido a la permanente remineralización local ${ }^{21}$, mejorando la resistencia a la placa, promocionando la neutralización del ácido en la boca, entre otros ${ }^{22-24}$.

Fuerza al cizallamiento. La microestructura homogénea de los giomeros y su carga alta de relleno, dan como resultado alta resistencia a la fuerza de cizalla, logrando estabilidad a largo plazo ${ }^{25}$, mejoras en las zonas de contracción, manipulación, pulido, resistencia a la compresión, etc., sobretodo frente al uso de un agente adhesivo de autograbado suave sobre el giomero antes del fraguado y a diferentes concentraciones de $\mathrm{pH}$ entre 2 y $2,2 .{ }^{10} \mathrm{Sin}$ embargo, no se mostró reducción significativa $(p>0,05)$ de esta propiedad ante un sistema adhesivo de distinta índole ${ }^{26}$.

Estabilidad del color. Los giomeros imitan la luz que refleja las propiedades de los dientes naturales, logrando mimetizar el color, ya que se fusionan de manera eficiente con la estructura dental circundante ${ }^{27}$. Los nanorellenos incluidos en los giomeros permiten un fácil pulido ${ }^{16,17}$, logrando alcanzar un brillo similar al esmalte natural ${ }^{28}$, mejorando aparentemente sus propiedades mecánicas ${ }^{8}$.

La estabilidad de color de los giomeros prácticamente no varía durante el modelado así como antes y después del curado; lo que asegura el acierto del color correcto incluso al hacer las capas ${ }^{29}$ e incluso podría ser usado en la parte anterior ${ }^{22}$. Sin embargo, se observó que después de 4 semanas de inmersión en bebidas como coca cola ( $\mathrm{pH} 3,0)$, jugo de naranja ( $\mathrm{pH} 3,88$ ) y leche bournvita $(\mathrm{pH} \mathrm{6,9)})^{30}$ aumentaba la rugosidad de la superficie del giomero y por consecuencia se daba la pérdida de la estabilidad del color ${ }^{31}$. 
Comportamiento clínico. Los giomeros tienen efecto terapeútico, proporcionando una barrera para proteger la dentina de los reactivos residuales que se difunden fuera de la restauración o de los fluidos orales que pueden penetrar la interfaz de la restauración con fugas ${ }^{32}$. Comisi afirma que los giomeros son muy resistentes, lo que le permite ser utilizado de manera muy eficaz en los dientes posteriores ${ }^{23}$; lo cual puede variar mostrando menos que el ideal en $11 \%$ de los $\operatorname{casos}^{33} \mathrm{y}$ a pesar de ello los giomeros tienen mejores resultados entre otros sistemas adhesivos en un promedio entre $88 \%$ y $100 \%{ }^{34}$.

\section{Discusión}

Los giomeros se recargan de iones de flúor de las fuentes externas como colutorios y pastas dentales que contengan flúor, como se demostró en el estudio de Gordan y cols. ${ }^{13}$, dándole una visión adicional a este fenómeno exposiciones en mayor concentración de fluoruros y de frecuencia.

La fuerza de cizalla no se ve afectada frente a sistemas adhesivos con recarga o no de flúor, así como también se ve mejores resultados con concentraciones de $\mathrm{pH}$ menos ácidos.

La estabilidad de color del giomero está sujeto a variaciones en el $\mathrm{pH}$ de las bebidas que incrementan la rugosidad y falta de mimetización con la estructura dentaria a tratar.

A pesar que los giomeros no presentan cambios marginales según calificaciones Alpha mayores a $90 \%$, por la menor contracción durante la polimerización ${ }^{35}$, esto puede alterarse según el tipo de restauración y el tiempo en el que es examinado principalmente como consecuencia de un contorno excesivo ${ }^{33}$.

Otras investigaciones demostraron después de tres y once ańos que las restauraciones con giomeros revelan integridad morfológica, funcional y mecánica comparable a la de resinas compuestas actuales ${ }^{36,37}$, tanto por su acabado en la superficie, por no mostrar decoloración marginal, ni sensibilidad postoperatoria o tinción en la superficie y por su buena adaptación marginal ${ }^{38}$.

Según la USPHS (United State Public Health Service), que es el único criterio usado por bastante tiempo para evaluar la durabilidad de las restauraciones, el cemento de ionómero vítreo modificado con resina (RMGIC) tiene menor flexibilidad (menor tendencia a la fractura), presenta restauraciones intactas con coincidencia de color y buena adaptación marginal en las superficies oclusales y proximales. Sólo que se observa una mancha oscura alrededor del margen cervical cuando hay pérdida parcial del giomero ${ }^{38}$.

\section{Conclusiones}

Comparando los resultados obtenidos en cada artículo en relación a las ventajas del uso de los giomeros en Odontopediatría, se pudo concluir que:
La exposición a fluoruros aumenta significativamente la liberación de flúor de los giomeros, sobretodo frente a dentífricos fluorados y aplicándolos dos veces al día, lo que asegura la remineralización de las piezas dentarias restauradas con dicho material.

Es recomendable el uso de los giomeros en zonas posteriores por la resistencia que presenta a la cizalla.

Al demostrar estabilidad al color y estética es posible usarlo en zonas anteriores.

Los giomeros demostraron buen comportamiento clínico, con adaptación marginal y efectividad en el control de caries.

$\mathrm{Si}$ bien, las restauraciones colocadas a los pacientes nińos a base de giomeros dan excelentes resultados, es necesario realizar estudios de seguimiento para evaluar su efectividad en el tiempo.

\section{Referencias bibliográficas}

1. La Importancia de la Salud Bucal [internet]. España: La República.es; c2017 [citado mayo 15 del 2017]. Disponible en: https://larepublica.es/2017/05/29/la-importancia-de-la-salud-bucal/

2. Ozdemir-Ozenen D, Sungurtekin E, Issever H, Sandalli N. Surface roughness of fluoride-releasing restorative materials after topical fluoride application. Eur J Paediatr Dent. 2013;14(1):68-72.

3. Día Mundial del futuro sin cavidades de Caries Dental [Internet]. Barcelona: Sociedad Española de Odontopediatría; c2019 [citado 2019 octubre 14]. Disponible en: https://www.odontologiapediatrica. com/2019/10/14/14-de-octubre-de-2019-dia-mundialdel-futuro-sin-cavidades-de-caries-dental/

4. Wilson A, Kent B. Glass Ionomers in Dentistry. 1ed. Chicago. Quintessence Publishing Co., 1989.

5. Rodríguez G, Pereira S. Evolución y tendencias actuales en resinas compuestas. Acta Odontol Venez. 2008;46(3):381-392.

6. Otazú C, Perona G. Técnica Restaurativa atraumática. Conceptos actuales. Rev Estomatol Herediana 2005;15(1):77-81.

7. Coutinho E, Cardoso MV, De Munck J, Neves AA, Van Landuyt KL, Poitevin A, et al. Bonding effectiveness and interfacial characterization of a nano-filled resin-modified glass-ionomer. Dent Mater. 2009; 25(11): 1347-57. DOI: 10.1016/j.dental.2009.06.004

8. Carvalho FG, Sampaio CS, Fucio SB, Carlo HL, Correr-Sobrinho L, Puppin-Rontani RM. Effect of chemical and mechanical degradation on surface roughness of three glass ionomers and a nanofilled resin composite. Oper Dent. 2012;37(5):509-17. DOI: 10.2341/10-406-L

9. Gil M, Sáenz M. Compómero: ¿Vidrio Ionomérico modificado con resina o resina modificada con vidrio ionomérico? Revisión de la literatura. Acta Odontol Venez 2001;39(1)57-60.

10. Kandaswamy D, Rajan KJ, Venkateshbabu N, Porkodi I. Shear bond strength evaluation of resin composite bonded to glass-ionomer cement using self-etching bonding 
agents with different $\mathrm{pH}$ : In vitro study. J Conserv Dent 2012;15(1):27-31. DOI: 10.4103/0972-0707.92602

11. Farah CS, Orton VG, Collard SM. Shear bond strength of chemical and light-cured glass ionomer cements bonded to resin composites. Aust Dent J 1998;43(2):81-6. DOI: $10.1111 /$ j.1834-7819.1998.tb06095.x.

12. Paredes J. Manejo odontológico preventivo, con aplicación de giomeros fluidos [Posgrado]. Universidad Nacional Mayor de San Marcos; 2017.

13. Gordan V, Mondragon E, Watson R, Garvan C, Mjör I. A clinical evaluation of a self-etching primer and a giomer restorative material Results at eight years. JADA. 2007 May; 138(5):621-7.DOI: 10.14219/jada.archive.2007.0233

14. Gopikrishna V, Abarajithan M, Krithikadatta J, Kandaswamy D. Shear bond strength evaluation of resin composite bonded to GIC using three different adhesives. Oper Dent. 2009;34(4):467-71. DOI: 10.2341/08009-L

15. Mathis RS, Ferracane JL. Properties of a glass-ionomer/ resin-composite hybrid material. Dent Mater. 1989; 5(5):355-8. DOI: 10.1016/0109-5641(89)90130-9

16. Kasraie S, Sokripour M, Safari M. Evaluation of micro-shear bond strength of resin modified glass-ionomer to composite resins using various bonding systems. J Conserv Dent. 2013;16(6):550-554. DOI: $10.4103 \% 2 F 0972-0707.120956$

17. Arora V, Kundabala M, Parolia A, Tomas M, Pai Viveknanda. Comparison of the shear bond strength of RMGIC to a resin composite using different adhesive systems: An in vitro study. J Conserv Dent. 2010;13(2):80-83. DOI: $10.4103 / 0972-0707.66716$

18. Dionysopoulos P, Kotsanos N y Pataridou A. Fluoride release and uptake by four new fluoride releasing restorative materials. J Oral Rehabil. 2003;30(9):866-72. DOI: 10.1046/j.1365-2842.2003.00993.x

19. Dhull KS, Nandlal B. Comparative evaluation of fluoride release from PRG-composites and compomer on application of topical fluoride: An in-vitro study. J Indian Soc Pedod Prev Dent 2009;27(1):27-32. DOI: 10.4103/0970-4388.50813

20. Bala O, Arisu HD, Yikilgan I, Arslan S, Gullu A. Evaluation of surface roughness and hardness of different glass ionomer cements. Eur J Dent. 2012;6(1):79-86. DOI: $10.1055 / s-0039-1698934$

21. Hiiri A, Ahovuo-Saloranta A, Nordblad A, Mäkelä M. Pit and fissure sealants versus fluoride varnishes for preventing dental decay in children and adolescents. Cochrane Database Syst Rev. 2010;17(3):CD003067. https://doi. org/10.1002/14651858.CD003067.pub3

22. Anusavice KJ, Zhang NZ, Shen C. Controlled release of chlorhexidine from UDMA-TEGDMA resin. J Dent Res. 2006;85(10):950-4. DOI: $10.1177 \% 2 \mathrm{~F} 154405910608501016$

23. Wiegand A, Buchalla W, Attin T. Review on fluoride-releasing restorative materials-fluoride release and uptake characteristics, antibacterial activity and influence on caries formation. Dent Mater. 2007;23(3):343-62. DOI: 10.1016/j.dental.2006.01.022
24. Nakajo K, Imazato S, Takahashi Y, Kiba W, Ebisu S, Takahashi N. Fluoride released from glass-ionomer cement is responsible to inhibit the acid production of caries-related oral streptococci. Dent Mater. 2009;25(6):703-8. DOI: $10.1016 /$ j.dental.2008.10.014

25. Ilda $Y$, Nikaida T, Kitayama S, Takagaki T, Inonue G, Ikeda M, et al. Evaluation of dentin bonding performance and acid-base resistance of the interface of two-step self-etching adhesive systems. Dent Mater J. 2009;28(4),493-500. DOI: 10.4012/dmj.28.493

26. Naoum S, O’Regan J, Ellakwa A, Benkhart R, Swain M, Martin E. The effect of repeated fluoride recharge and storage media on bond durability of fluoride rechargeable Giomer bonding agent. J Australian Dent. 2012;57(2):17883. DOI: $10.1111 /$ j.1834-7819.2012.01681.x

27. Korkmaz Y, Gurgan S, Firat E, Nathanson D. Shear bond strength of three different nano-restorative materials to dentin. Oper Dent. 2010;35(1):50-7. DOI: 10.2341/09-051-L

28. Han L, Okiji T. Evaluation of the ions release / incorporation of the prototype S-PRG filler-containing endodontic sealer, Dent Mater J. 2011;30(6):898-903. DOI: 10.4012/dmj.2011-101

29. Giomer/MiCD International Advisor Meeting in Kyoto. [internet]. Japan: Shofu inc; c2012 [citado 14 de julio 2012]. Disponible en: https://www.shofu.de/wp-content/uploads/2016/03/Giomer-Artikel-UK-2014-02MiCD.pdf

30. Hotwani K, Thosar N, Baliga S. Comparative in vitro assessment of color stability of hybrid esthetic restorative materials against various children's beverages. J Conserv Dent. 2014;17(1):70-74. DOI: 10.4103\%2F09720707.124154

31. Yousef M, Abo El Naga A. Color stability of different restoratives after exposure to coloring agents. J Am Sci. 2012;8(2):20-6.

32. Soubhagya M, Mallikarjun K, Deepak B, Thakur S, Nandini T, Arun J. Comparative in vitro evaluation of internal adaptation of resin-modified glass ionomer, flowable composite and bonding agent applied as a liner under composite restoration: A scanning electron microscope study. J Int Oral Health. 2015;7(4):27-31.

33. Wilson N, Gordan V, Brunton P, Wilson M, Crisp R, Mjor I. Two center evaluation of a resin composite/ self-etching restorative system: three-year findings. J Adhes Dent. 2006;8(1):47-51.

34. Peumans M, Kanumilli P, De Munck J, Van Landuyt K, Lambrechts P, Van Meerbeek B. Clinical effectiveness of contemporary adhesives: a systematic review of current clinical trials. Dent Mater. 2005;21(9):864-81. DOI: 10.1016/j.dental.2005.02.003

35. Manuja N, Pandit IK, Srivastava N, Gugnani N, Nagpal R. Comparative evaluation of shear bond strength of various esthetic restorative materials to dentin: an in vitro study. J Indian Soc Pedod Prev Dent. 2011;29(1):7-13. DOI: $10.4103 / 0970-4388.79913$

36. Abdel-Karim UM, El-Eraky M., Etman WM. Three-year clinical evaluation of two nano-hybrid giomer restorative composites. J Tanta Dent. 2014;11(3):213-22. DOI: 10.1016/j.tdj.2014.10.004 
37. Gordan VV, Blaser PK, Watson RE, Mjör IA, McEdward DL, Sensi LG, et al. A Clinical Evaluation of a Giomer Restorative System Containing Surface Prereacted Glass Ionomer Filler: Results From a 13-year Recall Examination. J Am Dent Assoc. 2014;145(10):1036-43. DOI: 10.14219/jada.2014.57
38. Jyothi KN, Annapurna S., Kumar AS, Venugopal P., Jayashankara CM Clinical Evaluation of Giomer- And Resin-Modified Glass Ionomer Cement in Class V Noncarious Cervical Lesions: An in Vivo Study. J Conserv Dent. 2011;14(4):409-13. DOI: 10.4103\%2F09720707.87214 
